\title{
Functional Encryption for Inner Product Predicates from Learning with Errors
}

\author{
Shweta Agrawal ${ }^{1, \star}$, David Mandell Freeman ${ }^{2, \star \star}$, and Vinod Vaikuntanathan ${ }^{3, \star \star \star}$ \\ ${ }^{1}$ University of California, Los Angeles, USA \\ shweta@cs.ucla.edu \\ ${ }^{2}$ Stanford University, USA \\ dfreemanecs.stanford.edu \\ 3 University of Toronto, Canada \\ vinodv@cs. toronto.edu
}

\begin{abstract}
We propose a lattice-based functional encryption scheme for inner product predicates whose security follows from the difficulty of the learning with errors (LWE) problem. This construction allows us to achieve applications such as range and subset queries, polynomial evaluation, and CNF/DNF formulas on encrypted data. Our scheme supports inner products over small fields, in contrast to earlier works based on bilinear maps.

Our construction is the first functional encryption scheme based on lattice techniques that goes beyond basic identity-based encryption. The main technique in our scheme is a novel twist to the identity-based encryption scheme of Agrawal, Boneh and Boyen (Eurocrypt 2010). Our scheme is weakly attribute hiding in the standard model.
\end{abstract}

Keywords: Functional encryption, predicate encryption, lattices, learning with errors.

\section{Introduction}

Traditional public-key encryption is "coarse," in the sense that any user in the system can decrypt only messages encrypted with that user's public key. In a line of research beginning with the work of Sahai and Waters [39], a number of researchers have asked how to make encryption more fine-grained. The result is the notion of functional encryption [16], in which secret keys allow users to learn functions of encrypted data. Two important examples of functional encryption are attribute-based encryption

* Part of this work done while at Microsoft Research Redmond. Research supported in part from a DARPA/ONR PROCEED award, and NSF grants 1118096, 1065276, 0916574 and 0830803.

${ }^{\star \star}$ Research supported by NSF and DARPA.

${ }^{\star \star}$ Part of this work done while at Microsoft Research Redmond. Supported by an NSERC Discovery grant and by DARPA under Agreement number FA8750-11-2-0225. The U.S. Government is authorized to reproduce and distribute reprints for Governmental purposes notwithstanding any copyright notation thereon. The views and conclusions contained herein are those of the authors and should not be interpreted as necessarily representing the official policies or endorsements, either expressed or implied, of DARPA or the U.S. Government. 
(ABE) [39, 27] and predicate encryption (PE) [17, 29]. In (key-policy) ABE and PE systems, each ciphertext $c$ is associated with an attribute $a$ and each secret key $s$ is associated with a predicate $f$. A user holding the key $s$ can decrypt $c$ if and only if $f(a)=1$. The difference between the two types of systems is in the amount of information revealed: an ABE system reveals the attribute associated with each ciphertext, while a PE system keeps the attribute hidden. (Formal definitions of these properties appear in Section 2, )

This hiding requirement has made predicate encryption systems much more difficult to construct than attribute-based encryption systems: while there exist ABE schemes that allow any access formula over attributes [35, 46], the most expressive PE scheme is that of Katz, Sahai, and Waters [29], who construct a PE scheme for inner product predicates. In such a scheme, attributes $a$ and predicates $f$ are expressed as vectors $\vec{v}_{a}$ and $\vec{w}_{f}$ respectively, and we say $f(a)=1$ if and only if $\left\langle\vec{v}_{a}, \vec{w}_{f}\right\rangle=0$. Despite this apparently restrictive structure, inner product predicates can support conjunction, subset and range queries on encrypted data [17] as well as disjunctions, polynomial evaluation, and CNF and DNF formulas [29].

All known constructions of attribute-based encryption [39, 27, 10, 21, 35, 26, 46, 8, 30, [34, 9] and predicate encryption [15, 1, 41, 17, 29, 42, 40, 33, 11, 30] make use of groups with bilinear maps, and the security of these schemes is based on many different, and often complex, assumptions. In particular, there is at present no known construction of predicate encryption for inner products based on a "standard" assumption in bilinear groups 1 As an example of a "nonstandard" assumption used in previous constructions, Katz, Sahai, and Waters present an assumption [29. Assumption 1] where the challenge consists of ten elements chosen in a specified way from a group whose order is the product of three large primes $p, q, r$, and the problem is to determine whether one of these elements has an order- $q$ component. While assumptions such as this one can often be shown to hold in a suitable "generic group model" (e.g., [29. Appendix A]), to obtain more confidence in security we would like to build ABE and PE schemes based on computational problems whose complexity is better understood.

Our Contribution. In this work we construct a lattice-based predicate encryption scheme for inner product predicates whose security follows from the difficulty of the learning with errors (LWE) problem. The LWE problem, in turn, is at least as hard as approximating the standard lattice problems GapSVP and SIVP in the worst case [38, 36] and is also conjectured to be difficult even for quantum adversaries. Our construction is the first functional encryption scheme based on lattice techniques that goes beyond basic identity-based encryption (which can be viewed as predicate encryption that tests equality on strings). Our construction is capable of instantiating all of the applications of predicate encryption proposed by Boneh and Waters [17] and Katz, Sahai, and Waters [29] 2] While our construction does not satisfy the strong notion of privacy defined by Katz, Sahai, and Waters [29], it does satisfy the slightly weaker notion considered by Okamoto and Takashima [33, 34] and Lewko et al. [30].

\footnotetext{
${ }^{1}$ Okamoto and Takashima [34] claim a PE construction from the decision linear assumption, but their paper only indicates how this is achieved for ABE.

${ }^{2}$ A detailed discussion of these applications can be found in the full version of this paper [2] §5].
} 


\subsection{Overview of the Construction}

Our Approach. Just as functional encryption in bilinear groups builds on the ideas and techniques introduced in constructions of identity-based encryption (IBE) in bilinear groups [14, 25, 12, 13, 44, 22], our construction builds on the ideas and techniques used to achieve identity-based encryption from the LWE assumption [24,5, 20,3,4]. However, there is a key difference between lattice IBE constructions (without random oracles) and bilinear-group constructions that makes this kind of generalization more difficult in the lattice setting. Namely, in the bilinear-group IBE constructions the groups remain fixed, while the ciphertexts and keys are manipulated so that group elements "cancel out" when a ciphertext matches a key. In the lattice IBE constructions, each key and ciphertext is constructed using a different lattice, and decryption only works when the key lattice and ciphertext lattice match. This structure does not easily generalize to the functional encryption setting, where each key may match many ciphertexts and each ciphertext may match many keys.

We solve this "lattice matching" problem using a new algebraic technique that builds on the IBE scheme of Agrawal, Boneh, and Boyen [3]. In our construction, we generate keys using a lattice $\Lambda_{f}$ that depends only on the predicate $f$, and we generate ciphertexts $c$ using a lattice $\Lambda_{a}$ that depends only on the attribute $a$. Given a ciphertext $c$ generated in this way and predicate $f$, we apply a suitable linear transformation that moves $c$ into the lattice $\Lambda_{f}$ if and only if $f(a)=1$. Once this transformation is applied, we can decrypt using a key associated with $\Lambda_{f}$.

The details of our scheme and security proof are in Section 4 To prove security, we use a simulation technique that draws on ideas introduced in [3]. In particular, we construct our simulation using a "punctured" trapdoor that allows the simulator to generate secret keys for any predicate $f$ such that $f(a)=0$, where $a$ is the "challenge" attribute. In the simulation we can use an LWE challenge to construct a ciphertext that either decrypts correctly or decrypts to a random message. While this technique suffices to prove that the system hides the message contents ("payload hiding"), it only allows us to prove a weak form of anonymity ("attribute hiding"). Specifically, given a ciphertext $c$ and a number of keys that do not decrypt $c$, the user cannot determine the attribute associated with $c$. In the strong form of attribute hiding, the user cannot determine the attribute associated with $c$ even when given keys that $d o$ decrypt $c$. (Formal definitions of these concepts appear in Section 2]) The weakened form of attribute hiding we do achieve is nonetheless more than is required for $\mathrm{ABE}$ and should be sufficient for many applications of PE.

Key Technical Ideas. Our encryption scheme is at its core based on the LWE scheme of Gentry, Peikert, and Vaikuntanathan [24, §7], which is itself a "dual" of the original Regev LWE scheme [38, §5]. From a geometric perspective, the public key in the GPV scheme describes a lattice $\Lambda$ used to construct ciphertexts, and the secret key is derived from the dual lattice $\Lambda^{\perp}$. Existing constructions of lattice-based IBE in the standard model [5, 20, 3, 4] use the GPV encryption scheme but replace the fixed lattice $\Lambda$ with a lattice $\Lambda_{\text {id }}$ that depends on the user's identity id. Decryption only works when the ciphertext lattice $\Lambda_{\mathrm{id}}$ and secret key lattice $\Lambda_{\text {id' }}$ are duals of each other, and there are several methods of ensuring that this is the case if and only if id $=\mathrm{id}^{\prime}$. 
In trying to adapt these constructions to the predicate encryption setting, we run into the problem that each ciphertext can be decrypted by many secret keys and each secret key can decrypt many ciphertexts. Thus we cannot require that key lattices match ciphertext lattices in the same way as above.

Before explaining our solution to this problem, let us recall the IBE scheme of Agrawal, Boneh, and Boyen [3]. In the ABB IBE scheme, the encryption lattice is constructed as

$$
\Lambda_{\mathrm{id}}=\Lambda_{q}\left(\mathbf{A}_{0} \| \mathbf{A}_{1}+H(\mathrm{id}) \mathbf{B}\right),
$$

where $\mathbf{A}_{0}, \mathbf{A}_{1}, \mathbf{B}$ are $n \times m$ matrices over $\mathbb{Z}_{q}$ and $H($ id) is a "full-rank difference" hash function. One can generate secret keys for $\Lambda_{\text {id }}^{\perp}$ using a short basis of $\Lambda_{q}^{\perp}\left(\mathbf{A}_{0}\right)$ and the basis extension technique of [5,20]. In the (selective-)security proof, the LWE challenge is embedded as the matrix $\mathbf{A}_{0}$, and the matrix $\mathbf{A}_{1}+H(\mathrm{id}) \mathbf{B}$ is equipped with a "punctured" trapdoor that allows the simulator to respond to secret key queries for all identities id not equal to the challenge identity id".

The algebraic structure of the ABB IBE scheme gives us the tools we need to solve the "lattice matching" problem described above. Specifically, in our predicate encryption scheme we encode an attribute vector $\vec{w}=\left(w_{1}, \ldots, w_{\ell}\right) \in \mathbb{Z}_{q}^{\ell}$ as the $n \times \ell m$ matrix

$$
\mathbf{B}_{\vec{w}}:=\left(w_{1} \mathbf{B}\|\cdots\| w_{\ell} \mathbf{B}\right) .
$$

where $\mathbf{B} \in \mathbb{Z}_{q}^{n \times m}$ is a uniformly random matrix chosen by the encryptor. We generate the ciphertext as a GPV encryption relative to the matrix

$$
\Lambda_{\vec{w}}:=\Lambda_{q}\left(\mathbf{A}_{0}\left\|\mathbf{A}_{1}+w_{1} \mathbf{B}\right\| \cdots \| \mathbf{A}_{\ell}+w_{\ell} \mathbf{B}\right)
$$

where the $\mathbf{A}_{i}$ are all $n \times m$ matrices. We view the ciphertext component that is close to $\Lambda_{\vec{w}}$ as a tuple $\left(\mathbf{c}_{0}, \ldots, \mathbf{c}_{\ell}\right) \in\left(\mathbb{Z}_{q}^{m}\right)^{\ell+1}$.

Since the recipient of a ciphertext does not know a priori which lattice was used to encrypt (indeed, this is exactly the anonymity property of predicate encryption), we cannot expect the recipient to possess a secret key derived from the dual of the ciphertext lattice as in the IBE case. Instead, we derive the key for a predicate vector $\vec{v}$ from the dual of a certain lattice $\Lambda_{\vec{v}}$ and apply a linear transformation $T_{\vec{v}}$ that moves the ciphertext into $\Lambda_{\vec{v}}$ exactly when $\langle\vec{v}, \vec{w}\rangle=0$. If this linear transformation is "short" (in the sense of not increasing the length of vectors too much), then a GPV secret key derived from $\Lambda_{\vec{v}}{ }^{\perp}$ can decrypt the ciphertext $T_{\vec{v}}(c)$.

Concretely, this transformation works as follows. For a predicate vector $\vec{v}=$ $\left(v_{1}, \ldots, v_{\ell}\right) \in \mathbb{Z}_{q}^{\ell}$, we define the linear transformation $T_{\vec{v}}:\left(\mathbb{Z}_{q}^{m}\right)^{\ell+1} \rightarrow \mathbb{Z}_{q}^{2 m}$ by

$$
T_{\vec{v}}\left(\mathbf{c}_{0}, \ldots, \mathbf{c}_{\ell}\right)=\left(\mathbf{c}_{0}, \sum_{i=1}^{\ell} v_{i} \mathbf{c}_{i}\right) .
$$

Some algebraic manipulation (detailed in Section 4) shows that applying this transformation to a ciphertext encrypted using $\Lambda_{\vec{w}}$ is equivalent to computing a GPV ciphertext using the lattice

$$
\Lambda_{\vec{v}, \vec{w}}:=\Lambda_{q}\left(\mathbf{A}_{0} \| \sum_{i=1}^{\ell} v_{i} \mathbf{A}_{i}+\langle\vec{v}, \vec{w}\rangle \mathbf{B}\right),
$$


Letting the secret key for $\vec{v}$ be the GPV secret key associated to $\Lambda_{q}^{\perp}\left(\mathbf{A}_{0} \| \sum_{i=1}^{\ell} v_{i} \mathbf{A}_{i}\right)$ allows the holder of a key for predicate $\vec{v}$ to decrypt a ciphertext associated with attribute $\vec{w}$ exactly when $\langle\vec{v}, \vec{w}\rangle=0$. In this aspect our construction is inspired by that of Katz, Sahai, and Waters [29]: the matrix B corresponds to the "masking terms" in a KSW ciphertext that "cancel out" exactly when $\langle\vec{v}, \vec{w}\rangle=0$.

The reader may have observed that in the above formulation, the requirement that the transformation $T_{\vec{v}}$ be "short" implies that we cannot use all vectors $\vec{v} \in \mathbb{Z}_{q}^{\ell}$ as predicates, but only ones whose entries have small absolute value (when viewed as integers in $(-q / 2, q / 2])$. In Section[4 we will show that decomposing the vector $\vec{v}$ into its binary representation enables our construction to use arbitrary vectors in $\mathbb{Z}_{q}^{\ell}$, at the expense of expanding the ciphertext by a factor of $\lg q$.

\section{Predicate Encryption}

We use the definition of predicate encryption proposed by Katz, Sahai, and Waters [29], which is based on the definition of searchable encryption proposed by Boneh and Waters [17]. We will let $n$ denote the security parameter throughout this paper.

Definition 2.1 ( [29, Definition 2.1]). A (key-policy) predicate encryption scheme for the class of predicates $\mathcal{F}$ over the set of attributes $\Sigma$ consists of four probabilistic polynomial-time algorithms Setup, KeyGen, Enc, Dec such that:

- Setup takes as input a security parameter $n$ and outputs a set of public parameters PP and a master secret key MK.

- KeyGen takes as input the master secret key MK and a (description of a) predicate $f \in \mathcal{F}$. It outputs a key sk .

- Enc takes as input the public parameters PP, an attribute $I \in \Sigma$, and a message $M$ in some associated message space $\mathcal{M}$. It returns a ciphertext $C$.

- Dec takes as input a secret key sk ${ }_{f}$ and a ciphertext $C$. It outputs either a message $M$ or the distinguished symbol $\perp$.

For correctness, we require that for all $n$, all (PP, MK) generated by $\operatorname{Setup}\left(1^{n}\right)$, all $f \in \mathcal{F}$, any key $\mathrm{sk}_{f} \leftarrow \operatorname{KeyGen}(\mathrm{MK}, f)$, all $I \in \Sigma$, and any ciphertext $C \leftarrow$ $\operatorname{Enc}(\mathrm{PP}, I, M)$ :

- If $f(I)=1$, then $\operatorname{Dec}\left(\mathrm{sk}_{f}, C\right)=M$.

- If $f(I)=0$, then $\operatorname{Dec}\left(\operatorname{sk}_{f}, C\right)=\perp$ with all but negligible probability.

In a ciphertext-policy scheme keys are associated with attributes and ciphertexts are associated with predicates; the syntax is otherwise the same.

Our construction in Section 4 satisfies a different correctness condition: If $f(I)=1$ and $C=\operatorname{Enc}(\mathrm{PP}, I, M)$, then $\operatorname{Dec}\left(\mathrm{sk}_{f}, C\right)=M$, but if $f(I)=0$ then $\operatorname{Dec}\left(\mathrm{sk}_{f}, C\right)$ is computationally indistinguishable from a uniformly random element in the message space $\mathcal{M}$. However, if $\mathcal{M}$ is exponentially large then we can easily transform our system into one satisfying Definition2.1 by restricting the message space to some subset $\mathcal{M}^{\prime} \subset$ $\mathcal{M}$ with $\left|\mathcal{M}^{\prime}\right| /|\mathcal{M}|=\operatorname{negl}(n)$. 


\subsection{Security}

There are several notions of security for predicate encryption schemes. The most basic is payload hiding, which guarantees that no efficient adversary can obtain any information about the encrypted message, but allows information about attributes to be revealed. A stronger notion is attribute hiding, which guarantees in addition that no efficient adversary can obtain any information about the attribute associated with a ciphertext. Following Lewko et al. [30, Definition 17], we also define an intermediate notion, weak attribute hiding, which makes the same guarantee only in the case that the adversary cannot decrypt the ciphertext. Our definition of security is "selective," in the sense that the adversary must commit to its challenge attributes before seeing any secret keys.

Definition 2.2 ( [29, Definition 2.2]). A predicate encryption scheme with respect to $\mathcal{F}$ and $\Sigma$ is attribute hiding if for all probabilistic polynomial-time adversaries $\mathcal{A}$, the advantage of $\mathcal{A}$ in the following experiment is negligible in the security parameter $n$ :

1. $\mathcal{A}\left(1^{n}\right)$ outputs $I_{0}, I_{1} \in \Sigma$.

2. Setup $\left(1^{n}\right)$ is run to generate PP and MK, and the adversary is given PP.

3. $\mathcal{A}$ may adaptively request keys for any predicates $f_{1}, \ldots, f_{\ell} \in \mathcal{F}$ subject to the restriction that $f_{i}\left(I_{0}\right)=f_{i}\left(I_{1}\right)$ for all $i$. In response, $\mathcal{A}$ is given the corresponding keys sk $f_{i} \leftarrow$ KeyGen $\left(\mathrm{MK}, f_{i}\right)$.

4. $\mathcal{A}$ outputs two equal-length messages $M_{0}, M_{1}$. If there is an $i$ for which $f_{i}\left(I_{0}\right)=$ $f_{i}\left(I_{1}\right)=1$, then it is required that $M_{0}=M_{1}$. A random bit $b$ is chosen, and $\mathcal{A}$ is given the ciphertext $C \leftarrow \operatorname{Enc}\left(\mathrm{PP}, I_{b}, M_{b}\right)$.

5. The adversary may continue to request keys for additional predicates, subject to the same restrictions as before.

6. $\mathcal{A}$ outputs a bit $b^{\prime}$, and succeeds if $b^{\prime}=b$. The advantage of $\mathcal{A}$ is the absolute value of the difference between its success probability and $1 / 2$.

We say the scheme is weakly attribute hiding if the same condition holds for adversaries $\mathcal{A}$ that are only allowed to request keys for predicates $f_{i}$ with $f_{i}\left(I_{0}\right)=$ $f_{i}\left(I_{1}\right)=0$. We say the scheme is payload hiding if we require $I_{0}=I_{1}$.

We observe that any scheme that is attribute hiding is weakly attribute hiding, and any scheme that is weakly attribute hiding is payload hiding. (In the payload hiding game no adversary can achieve nonzero advantage when requesting a key for a predicate $f$ with $f\left(I_{0}\right)=f\left(I_{1}\right)=1$, so we may assume without loss of generality that the adversary does not request such a key.)

Remark 2.3. In our construction the spaces $\mathcal{F}$ of predicates and $\Sigma$ of attributes depend on the public parameters PP output by Setup. We thus modify the security game so as to give the adversary descriptions of $\mathcal{F}$ and $\Sigma$ before Step 1 and run the remainder of the game (including any remaining steps in the Setup algorithm) as described.

\section{Lattice Preliminaries}

In this section we collect the results from the literature that we will need for our construction and the proof of security. 
Notation. For any integer $q \geq 2$, we let $\mathbb{Z}_{q}$ denote the ring of integers modulo $q$ and we represent $\mathbb{Z}_{q}$ as integers in $(-q / 2, q / 2]$. We let $\mathbb{Z}_{q}^{n \times m}$ denote the set of $n \times m$ matrices with entries in $\mathbb{Z}_{q}$. We use bold capital letters (e.g. A) to denote matrices, bold lowercase letters (e.g. $\mathbf{x}$ ) to denote vectors that are components of our encryption scheme, and arrows (e.g. $\vec{v}$ ) to denote vectors that represent attributes or predicates. The notation $\mathbf{A}^{\top}$ denotes the transpose of the matrix $\mathbf{A}$. When we say a matrix defined over $\mathbb{Z}_{q}$ has full rank, we mean that it has full rank modulo each prime factor of $q$. The notation $\lfloor x\rceil$ denotes the nearest integer to $x$, rounding towards 0 for half-integers.

\subsection{Lattices}

An $m$-dimensional lattice $\Lambda$ is a full-rank discrete subgroup of $\mathbb{R}^{m}$. A basis of $\Lambda$ is a linearly independent set of vectors whose span is $\Lambda$. We will usually be concerned with integer lattices, i.e., those whose points have coordinates in $\mathbb{Z}^{m}$. Among these lattices are the " $q$-ary" lattices defined as follows: for any integer $q \geq 2$ and any $\mathbf{A} \in \mathbb{Z}_{q}^{n \times m}$, we define

$$
\begin{aligned}
& \Lambda_{q}^{\perp}(\mathbf{A}):=\left\{\mathbf{e} \in \mathbb{Z}^{m}: \mathbf{A} \cdot \mathbf{e}=\mathbf{0} \bmod q\right\} \\
& \Lambda_{q}^{\mathbf{u}}(\mathbf{A}):=\left\{\mathbf{e} \in \mathbb{Z}^{m}: \mathbf{A} \cdot \mathbf{e}=\mathbf{u} \bmod q\right\} .
\end{aligned}
$$

The lattice $\Lambda_{q}^{\mathbf{u}}(\mathbf{A})$ is a coset of $\Lambda_{q}^{\perp}(\mathbf{A})$; namely, $\Lambda_{q}^{\mathbf{u}}(\mathbf{A})=\Lambda_{q}^{\perp}(\mathbf{A})+\mathbf{t}$ for any $\mathbf{t}$ such that $\mathbf{A} \cdot \mathbf{t}=\mathbf{u} \bmod q$.

The Gram-Schmidt norm of a basis. Let $\mathbf{S}=\left\{\mathbf{s}_{1}, \ldots, \mathbf{s}_{k}\right\}$ be a set of vectors in $\mathbb{R}^{m}$. We use the following standard notation:

- $\|\mathbf{S}\|$ denotes the length of the longest vector in $\mathbf{S}$, i.e., $\max _{1 \leq i \leq k}\left\|\mathbf{s}_{i}\right\|$.

- $\widetilde{\mathbf{S}}:=\left\{\tilde{\mathbf{s}}_{1}, \ldots, \tilde{\mathbf{s}}_{k}\right\} \subset \mathbb{R}^{m}$ denotes the Gram-Schmidt orthogonalization of the vectors $\mathbf{s}_{1}, \ldots, \mathbf{s}_{k}$.

We refer to $\|\widetilde{\mathbf{S}}\|$ as the Gram-Schmidt norm of $\mathbf{S}$.

Ajtai [6] and later Alwen and Peikert [7] showed how to sample an almost uniform matrix $\mathbf{A} \in \mathbb{Z}_{q}^{n \times m}$ along with a basis $\mathbf{S}$ of $\Lambda_{q}^{\perp}(\mathbf{A})$ with low Gram-Schmidt norm.

Theorem 3.1 ( [7, Theorem 3.2] with $\delta=1 / 3$ ). Let $q, n, m$ be positive integers with $q \geq 2$ and $m \geq 6 n \lg q$. There is a probabilistic polynomial-time algorithm $\operatorname{TrapGen}(q, n, m)$ that with overwhelming probability (in $n)$ outputs a pair $(\mathbf{A} \in$ $\left.\mathbb{Z}_{q}^{n \times m}, \mathbf{S} \in \mathbb{Z}^{m \times m}\right)$ such that $\mathbf{A}$ is statistically close to uniform in $\mathbb{Z}_{q}^{n \times m}$ and $\mathbf{S}$ is a basis for $\Lambda_{q}^{\perp}(\mathbf{A})$ satisfying

$$
\|\widetilde{\mathbf{S}}\| \leq O(\sqrt{n \log q}) \text { and } \quad\|\mathbf{S}\| \leq O(n \log q) .
$$

Gaussian Distributions. Let $L$ be a discrete subset of $\mathbb{Z}^{n}$. For any vector $\mathbf{c} \in \mathbb{R}^{n}$ and any positive parameter $\sigma \in \mathbb{R}_{>0}$, let $\rho_{\sigma, \mathbf{c}}(\mathbf{x}):=\exp \left(-\pi\|\mathbf{x}-\mathbf{c}\|^{2} / \sigma^{2}\right)$ be the Gaussian function on $\mathbb{R}^{n}$ with center $\mathbf{c}$ and parameter $\sigma$. Let $\rho_{\sigma, \mathbf{c}}(L):=\sum_{\mathbf{x} \in L} \rho_{\sigma, \mathbf{c}}(\mathbf{x})$ be the discrete integral of $\rho_{\sigma, \mathbf{c}}$ over $L$ (which always converges), and let $\mathcal{D}_{L, \sigma, \mathbf{c}}$ be the discrete Gaussian distribution over $L$ with center $\mathbf{c}$ and parameter $\sigma$. Specifically, for 
all $\mathbf{y} \in L$, we have $\mathcal{D}_{L, \sigma, \mathbf{c}}(\mathbf{y})=\frac{\rho_{\sigma, \mathbf{c}}(\mathbf{y})}{\rho_{\sigma, \mathbf{c}}(L)}$. For notational convenience, $\rho_{\sigma, \mathbf{0}}$ and $\mathcal{D}_{L, \sigma, \mathbf{0}}$ are abbreviated as $\rho_{\sigma}$ and $\mathcal{D}_{L, \sigma}$, respectively.

The following lemma gives a bound on the length of vectors sampled from a discrete Gaussian. The result follows from [32, Lemma 4.4], using [24, Lemma 5.3] to bound the smoothing parameter.

Lemma 3.2. Let $\Lambda$ be an $n$-dimensional lattice, let $\mathbf{T}$ be a basis for $\Lambda$, and suppose $\sigma \geq\|\widetilde{\mathbf{T}}\| \cdot \omega(\sqrt{\log n})$. Then for any $\mathbf{c} \in \mathbb{R}^{n}$ we have

$$
\operatorname{Pr}\left[\|\mathbf{x}-\mathbf{c}\|>\sigma \sqrt{n}: \mathbf{x} \stackrel{\mathrm{R}}{\leftarrow} \mathcal{D}_{\Lambda, \sigma, \mathbf{c}}\right] \leq \operatorname{negl}(n)
$$

\subsection{Sampling Algorithms}

We will use the following algorithms to sample short vectors from specific lattices. Looking ahead, the algorithm SampleLeft [3, 20] will be used to sample keys in the real system, while the algorithm SampleRight [3] will be used to sample keys in the simulation.

Algorithm SampleLeft $\left(\mathbf{A}, \mathbf{B}, \mathbf{T}_{\mathbf{A}}, \mathbf{u}, \sigma\right)$ :

Inputs: a full rank matrix $\mathbf{A}$ in $\mathbb{Z}_{q}^{n \times m}$, a "short" basis $\mathbf{T}_{\mathbf{A}}$ of $\Lambda_{q}^{\perp}(\mathbf{A})$, a matrix $\mathbf{B}$ in $\mathbb{Z}_{q}^{n \times m_{1}}$, a vector $\mathbf{u} \in \mathbb{Z}_{q}^{n}$, and a Gaussian parameter $\sigma$.

Output: Let $\mathbf{F}:=(\mathbf{A} \| \mathbf{B})$. The algorithm outputs a vector $\mathbf{e} \in \mathbb{Z}^{m+m_{1}}$ in the coset $\Lambda_{q}^{\mathbf{u}}(\mathbf{F})$.

Theorem 3.3 ( [3, Theorem 17], [20, Lemma 3.2]). Let $q>2, m>n$ and $\sigma>$ $\left\|\widetilde{\mathbf{T}_{\mathbf{A}}}\right\| \cdot \omega\left(\sqrt{\log \left(m+m_{1}\right)}\right)$. Then SampleLeft $\left(\mathbf{A}, \mathbf{B}, \mathbf{T}_{\mathbf{A}}, \mathbf{u}, \sigma\right)$ taking inputs as in (3.1) outputs a vector $\mathbf{e} \in \mathbb{Z}^{m+m_{1}}$ distributed statistically close to $\mathcal{D}_{\Lambda_{q}^{u}(\mathbf{F}), \sigma}$, where $\mathbf{F}:=$ $(\mathbf{A} \| \mathbf{B})$.

Algorithm SampleRight $\left(\mathbf{A}, \mathbf{B}, \mathbf{R}, \mathbf{T}_{\mathbf{B}}, \mathbf{u}, \sigma\right)$ :

Inputs: matrices $\mathbf{A}$ in $\mathbb{Z}_{q}^{n \times k}$ and $\mathbf{R}$ in $\mathbb{Z}^{k \times m}$, a full rank matrix $\mathbf{B}$ in $\mathbb{Z}_{q}^{n \times m}$, a "short" basis $\mathbf{T}_{\mathbf{B}}$ of $\Lambda_{q}^{\perp}(\mathbf{B})$, a vector $\mathbf{u} \in \mathbb{Z}_{q}^{n}$, and a Gaussian parameter $\sigma$.

Output: Let $\mathbf{F}:=(\mathbf{A} \| \mathbf{A R}+\mathbf{B})$. The algorithm outputs a vector $\mathbf{e} \in \mathbb{Z}^{m+k}$ in the coset $\Lambda_{q}^{\mathbf{u}}(\mathbf{F})$.

Often the matrix $\mathbf{R}$ given to the algorithm as input will be a random matrix in $\{1,-1\}^{m \times m}$. Let $S^{m}$ be the $m$-sphere $\left\{\mathbf{x} \in \mathbb{R}^{m+1}:\|\mathbf{x}\|=1\right\}$. We define $s_{R}:=\|\mathbf{R}\|$ $:=\sup _{\mathbf{x} \in S^{m-1}}\|\mathbf{R} \cdot \mathbf{x}\|$.

Theorem 3.4 ( [3, Theorem 19]). Let $q>2, m>n$ and $\sigma>\left\|\widetilde{\mathbf{T}_{\mathbf{B}}}\right\| \cdot s_{R} \cdot \omega(\sqrt{\log m})$. Then SampleRight $\left(\mathbf{A}, \mathbf{B}, \mathbf{R}, \mathbf{T}_{\mathbf{B}}, \mathbf{u}, \sigma\right)$ taking inputs as in (3.2) outputs a vector $\mathbf{e} \in$ $\mathbb{Z}^{m+k}$ distributed statistically close to $\mathcal{D}_{\Lambda_{q}^{\mathrm{u}}(\mathbf{F}), \sigma}$, where $\mathbf{F}:=(\mathbf{A} \| \mathbf{A R}+\mathbf{B})$. 


\subsection{The LWE Problem}

The learning with errors problem, or LWE, is the problem of determining a secret vector over $\mathbb{Z}_{q}$ given an arbitrary number of "noisy" inner products. The decision variant is to distinguish such samples from random. More formally, we define the (average-case) problem as follows:

Definition 3.5 ( [38] $)$. Let $n \geq 1$ and $q \geq 2$ be integers, and let $\chi$ be a probability distribution on $\mathbb{Z}_{q}$. For $\mathbf{s} \in \mathbb{Z}_{q}^{n}$, let $A_{\mathbf{s}, \chi}$ be the probability distribution on $\mathbb{Z}_{q}^{n} \times \mathbb{Z}_{q}$ obtained by choosing a vector $\mathbf{a} \in \mathbb{Z}_{q}^{n}$ uniformly at random, choosing $e \in \mathbb{Z}_{q}$ according to $\chi$, and outputting $(\mathbf{a},\langle\mathbf{a}, \mathbf{s}\rangle+e)$.

(a) The search-LWE $\mathrm{LW}_{q, \chi}$ problem is: for uniformly random $\mathbf{s} \in \mathbb{Z}_{q}^{n}$, given a $\operatorname{poly}(n)$ number of samples from $A_{\mathbf{s}, \chi}$, output s.

(b) The decision- $\mathrm{LWE}_{q, n, \chi}$ problem is: for uniformly random $\mathbf{s} \in \mathbb{Z}_{q}^{n}$, given a poly $(n)$ number of samples that are either (all) from $A_{\mathbf{s}, \chi}$ or (all) uniformly random in $\mathbb{Z}_{q}^{n} \times \mathbb{Z}_{q}$, output 0 if the former holds and 1 if the latter holds.

We say the decision- $\operatorname{LWE}_{q, n, \chi}$ problem is infeasible if for all polynomial-time algorithms $\mathcal{A}$, the probability that $\mathcal{A}$ solves the decision-LWE problem (over $\mathbf{s}$ and $\mathcal{A}$ 's random coins) is negligibly close to $1 / 2$ as a function of $n$.

The power of the LWE problem comes from the fact that for certain noise distributions $\chi$, solving the search-LWE problem is as hard as finding approximate solutions to the shortest independent vectors problem (SIVP) and the decision version of the shortest vector problem (GapSVP) in the worst case. For polynomial size $q$ there is a quantum reduction due to Regev, while for exponential size $q$ there is a classical reduction due to Peikert. Furthermore, the search and decision versions of the problem are equivalent whenever $q$ is a product of small primes. These results are summarized in the following:

Definition 3.6. For $\alpha \in(0,1)$ and an integer $q>2$, let $\bar{\Psi}_{\alpha}$ denote the probability distribution over $\mathbb{Z}_{q}$ obtained by choosing $x \in \mathbb{R}$ according to the normal distribution with mean 0 and standard deviation $\alpha / \sqrt{2 \pi}$ and outputting $\lfloor q x\rceil$.

Theorem 3.7 ( [38]). Let $n, q$ be integers and $\alpha \in(0,1)$ such that $q=\operatorname{poly}(n)$ and $\alpha q>2 \sqrt{n}$. If there exists an efficient (possibly quantum) algorithm that solves decision$\mathrm{LWE}_{q, n, \bar{\Psi}_{\alpha}}$, then there exists an efficient quantum algorithm that approximates SIVP and GapSVP to within $\tilde{O}(n / \alpha)$ in the worst case.

Theorem 3.8 ( [36]). Let $n, q$ be integers and $\alpha \in(0,1)$, and $q=\prod_{i} q_{i} \geq 2^{n / 2}$, where the $q_{i}$ are distinct primes satisfying $\omega(\sqrt{\log n}) / \alpha \leq q_{i} \leq \operatorname{poly}(n)$. If there exists an efficient (classical) algorithm that solves decision- $\mathrm{LWE}_{q, n}, \bar{\Psi}_{\alpha}$, then there exists an efficient (classical) algorithm that approximates GapSVP to within $\tilde{O}(n / \alpha)$ in the worst case.

The following lemma will be used to show correctness of decryption.

Lemma 3.9 ( [3, Lemma 12]). Let e be some vector in $\mathbb{Z}^{m}$ and let $\mathbf{y} \leftarrow \bar{\Psi}_{\alpha}^{m}$. Then the quantity $|\langle\mathbf{e}, \mathbf{y}\rangle|$ when treated as an integer in $(-q / 2, q / 2]$ satisfies

$$
|\langle\mathbf{e}, \mathbf{y}\rangle| \leq\|\mathbf{e}\| q \alpha \cdot \omega(\sqrt{\log m})+\|\mathbf{e}\| \sqrt{m} / 2
$$

with overwhelming probability (in $\mathrm{m}$ ). 


\section{A Functional Encryption Scheme for Inner Product Predicates}

In our system, each secret key will be associated with a predicate vector $\vec{v} \in \mathbb{Z}_{q}^{\ell}$ (for some fixed $\ell \geq 2$ ) and each ciphertext will be associated with an attribute vector $\vec{w} \in \mathbb{Z}_{q}^{\ell}$. Decryption should succeed if and only if $\langle\vec{v}, \vec{w}\rangle=0(\bmod q)$. Hence the predicate associated with the secret key is defined as $f_{\vec{v}}(\vec{w})=1$ if $\langle\vec{v}, \vec{w}\rangle=0(\bmod q)$, and $f_{\vec{v}}(\vec{w})=0$ otherwise.

\subsection{The Construction}

Let $n \in \mathbb{Z}^{+}$be a security parameter and $\ell$ be the dimension of predicate and attribute vectors. Let $q=q(n, \ell)$ and $m=m(n, \ell)$ be positive integers. Let $\sigma=\sigma(n, \ell)$ and $\alpha=\alpha(n, \ell)$ be positive real Gaussian parameters. Define $k=k(n, \ell):=\lfloor\lg q\rfloor$. The encryption scheme described below encrypts a single bit; we show how to encrypt multiple bits in the full version of this paper [2, §4.5].

LinFE.Setup $\left(1^{n}, 1^{\ell}\right)$ : On input a security parameter $n$ and a parameter $\ell$ denoting the dimension of predicate and attribute vectors, do:

1. Use the algorithm $\operatorname{TrapGen}(q, n, m$ ) (from Theorem 3.1) to select a matrix $\mathbf{A} \in \mathbb{Z}_{q}^{n \times m}$ together with a full-rank set of vectors $\mathbf{T}_{\mathbf{A}} \subseteq \Lambda_{q}^{\perp}(\mathbf{A})$ such that $\left\|\widetilde{\mathbf{T}_{\mathbf{A}}}\right\| \leq m \cdot \omega(\sqrt{\log m})$.

2. Choose $\ell \cdot(1+k)$ uniformly random matrices $\mathbf{A}_{i, \gamma} \in \mathbb{Z}_{q}^{n \times m}$ for $i=1, \ldots, \ell$ and $\gamma=0, \ldots, k$.

3. Select a uniformly random vector $\mathbf{u} \in \mathbb{Z}_{q}^{n}$.

Output PP $=\left(\mathbf{A},\left\{\mathbf{A}_{i, \gamma}\right\}_{i \in\{1, \ldots, \ell\}, \gamma \in\{0, \ldots, k\}}, \mathbf{u}\right)$ and $\mathrm{MK}=\mathbf{T}_{\mathbf{A}}$.

LinFE.KeyGen(PP, MK, $\vec{v})$ : On input public parameters $\mathrm{PP}$, a master secret key MK, and a predicate vector $\vec{v}=\left(v_{1}, \ldots, v_{\ell}\right) \in \mathbb{Z}_{q}^{\ell}$, do:

1. For $i=1, \ldots, \ell$, let $\hat{v}_{i}$ be the integer in $[0, q-1]$ congruent to $v_{i} \bmod q$. Write the binary decomposition of $\hat{v}_{i}$ as

$$
\hat{v}_{i}=\sum_{\gamma=0}^{k} v_{i, \gamma} \cdot 2^{\gamma},
$$

where $v_{i, \gamma}$ are in $\{0,1\}$.

2. Define the matrices

$$
\begin{aligned}
\mathbf{C}_{\vec{v}} & :=\sum_{i=1}^{\ell} \sum_{\gamma=0}^{k} v_{i, \gamma} \mathbf{A}_{i, \gamma} \quad \in \mathbb{Z}_{q}^{n \times m}, \\
\mathbf{A}_{\vec{v}} & :=\left[\mathbf{A} \| \mathbf{C}_{\vec{v}}\right] \quad \in \mathbb{Z}_{q}^{n \times 2 m} .
\end{aligned}
$$

3. Using the master secret key $M K=\left(\mathbf{T}_{\mathbf{A}}, \sigma\right)$, compute $\mathbf{e} \leftarrow$ SampleLeft $\left(\mathbf{A}, \mathbf{C}_{\vec{v}}\right.$, $\left.\mathbf{T}_{\mathbf{A}}, \mathbf{u}, \sigma\right)$.

Then $\mathbf{e}$ is a vector in $\mathbb{Z}^{2 m}$ satisfying $\mathbf{A}_{\vec{v}} \cdot \mathbf{e}=\mathbf{u} \bmod q$.

Output the secret key $\mathrm{sk}_{\vec{v}}=\mathbf{e}$. 
LinFE.Enc $(\mathrm{PP}, \vec{w}, M)$ : On input public parameters $\mathrm{PP}$, an attribute vector $\vec{w}$, and a message $M \in\{0,1\}$, do:

1. Choose a uniformly random matrix $\mathbf{B} \stackrel{R}{\leftarrow} \mathbb{Z}_{q}^{n \times m}$.

2. Choose a uniformly random $\mathrm{s} \stackrel{\mathrm{R}}{\leftarrow} \mathbb{Z}_{q}^{n}$.

3. Choose a noise vector $\mathbf{x} \leftarrow \bar{\Psi}_{\alpha}^{m}$ and a noise term $x \leftarrow \bar{\Psi}_{\alpha}$.

4. Compute $\mathbf{c}_{0} \leftarrow \mathbf{A}^{\top} \mathbf{s}+\mathbf{x} \in \mathbb{Z}_{q}^{m}$.

5. For $i=1, \ldots, \ell$ and $\gamma=0, \ldots, k$, do the following:

(a) Pick a random matrix $\mathbf{R}_{i, \gamma} \in\{-1,1\}^{m \times m}$.

(b) Compute $\mathbf{c}_{i, \gamma} \leftarrow\left(\mathbf{A}_{i, \gamma}+2^{\gamma} w_{i} \mathbf{B}\right)^{\top} \mathbf{s}+\mathbf{R}_{i, \gamma}^{\top} \mathbf{x} \quad \in \mathbb{Z}_{q}^{m}$.

6. Compute $c^{\prime} \leftarrow \mathbf{u}^{\top} \mathbf{s}+x+M \cdot\lfloor q / 2\rceil \in \mathbb{Z}_{q}$.

Output the ciphertext $\mathrm{CT}:=\left(\mathbf{c}_{0},\left\{\mathbf{c}_{i, \gamma}\right\}_{i \in\{1, \ldots, \ell\}, \gamma \in\{0, \ldots, k\}}, c^{\prime}\right)$.

LinFE.Dec $\left(\mathrm{PP}, \mathrm{sk}_{\vec{v}}, \mathrm{CT}\right)$ : On input public parameters $\mathrm{PP}$, a secret key $\mathrm{sk}_{\vec{v}}$ for predicate vector $\vec{v}$, and a ciphertext $\mathrm{CT}=\left(\mathbf{c}_{0},\left\{\mathbf{c}_{i, \gamma}\right\}_{i \in\{1, \ldots, \ell\}, \gamma \in\{0, \ldots, k\}}, c^{\prime}\right)$, do:

1. Define the binary expansion of the vector $\vec{v}$ as in (4.1) and compute

$$
\mathbf{c}_{\vec{v}}:=\sum_{i=1}^{\ell} \sum_{\gamma=0}^{k} v_{i, \gamma} \mathbf{c}_{i, \gamma} .
$$

2. Let $\mathbf{c}:=\left[\mathbf{c}_{0} \mid \mathbf{c}_{\vec{v}}\right]$.

3. Compute $z \leftarrow c^{\prime}-\mathbf{e}^{\top} \mathbf{c}(\bmod q)$.

Output 0 if $|z|<q / 4$ (when interpreted as in integer in $(-q / 2, q / 2]$ ) and 1 otherwise.

For consistency with prior work, we choose the noise in Step 3 of Enc from the rounded continuous Gaussian $\bar{\Psi}_{\alpha}$. It was pointed out to us by a referee that one can instead use the discrete Gaussian $\mathcal{D}_{\mathbb{Z}, \alpha q}$ and obtain a system with the same security guarantee (up to a factor of $\sqrt{2}$ ); this result follows from [28, Lemma 2], using the work of Peikert [37].

\subsection{Correctness}

We now show that for certain parameter choices, if a bit $M$ is encrypted to the attribute vector $\vec{w}$, the secret key $\mathbf{s}_{\vec{v}}$ corresponds to a predicate vector $\vec{v}$, and $\langle\vec{v}, \vec{w}\rangle=0(\bmod q)$, then the LinFE.Dec algorithm recovers $M$.

Lemma 4.1. Suppose the parameters $q$ and $\alpha$ are such that

$$
q / \lg q=\Omega\left(\sigma \cdot \ell \cdot m^{3 / 2}\right) \quad \text { and } \quad \alpha \leq(\log q \cdot \sigma \cdot \ell \cdot m \cdot \omega \sqrt{\log m})^{-1} .
$$

Let $\mathbf{e} \leftarrow \operatorname{KeyGen}(\mathrm{PP}, \mathrm{MK}, \vec{v}), \mathrm{CT} \leftarrow \operatorname{Enc}(\mathrm{PP}, \vec{w}, M)$, and $\tilde{M} \leftarrow \operatorname{Dec}(\mathrm{PP}, \mathbf{e}, \mathrm{CT})$. If $\langle\vec{v}, \vec{w}\rangle=0(\bmod q)$, then with overwhelming probability we have $M^{\prime}=M$.

Proof. During the first step of LinFE.Dec we compute $\mathbf{c}_{\vec{v}}$, which is by definition:

$$
\mathbf{c}_{\vec{v}}=\sum_{i=1}^{\ell} \sum_{\gamma=0}^{k} v_{i, \gamma} \mathbf{c}_{i, \gamma} .
$$


This can be expanded as

$$
\begin{aligned}
\mathbf{c}_{\vec{v}} & =\sum_{i=1}^{\ell} \sum_{\gamma=0}^{k} v_{i, \gamma}\left[\left(\mathbf{A}_{i, \gamma}+2^{\gamma} w_{i} \mathbf{B}\right)^{\top} \mathbf{s}+\mathbf{R}_{i, \gamma}^{\top} \mathbf{x}\right] \\
& =\left(\sum_{i=1}^{\ell} \sum_{\gamma=0}^{k} v_{i, \gamma} \mathbf{A}_{i, \gamma}\right)^{\top} \mathbf{s}+\underbrace{\left(\sum_{i=1}^{\ell} \sum_{\gamma=0}^{k} 2^{\gamma} v_{i, \gamma} w_{i}\right)}_{\langle\vec{v}, \vec{w}\rangle(\bmod q)} \mathbf{B}^{\top} \mathbf{s}+\sum_{i=1}^{\ell} \sum_{\gamma=0}^{k} v_{i, \gamma} \mathbf{R}_{i, \gamma}^{\top} \mathbf{x} .
\end{aligned}
$$

If $\langle\vec{v}, \vec{w}\rangle=0(\bmod q)$, then the middle term of (4.2) disappears, leaving

$$
\mathbf{c}_{\vec{v}}=\left(\sum_{i=1}^{\ell} \sum_{\gamma=0}^{k} v_{i, \gamma} \mathbf{A}_{i, \gamma}\right)^{\top} \mathbf{s}+\sum_{i=1}^{\ell} \sum_{\gamma=0}^{k} v_{i, \gamma} \mathbf{R}_{i, \gamma}^{\top} \mathbf{x} \quad(\bmod q) .
$$

In the second step of LinFE.Dec we have:

$$
\begin{aligned}
\mathbf{c}=\left[\mathbf{c}_{0} \mid \mathbf{c}_{\vec{v}}\right] & =\left[\mathbf{A} \| \sum_{i=1}^{\ell} \sum_{\gamma=0}^{k} v_{i, \gamma} \mathbf{A}_{i, \gamma}\right]^{\top} \mathbf{s}+\left[\mathbf{x} \mid \sum_{i=1}^{\ell} \sum_{\gamma=0}^{k} v_{i, \gamma} \mathbf{R}_{i, \gamma}^{\top} \mathbf{x}\right] \quad(\bmod q) \\
& =\mathbf{A}_{\vec{v}}^{\top} \cdot \mathbf{s}+\left[\mathbf{x} \mid \sum_{i=1}^{\ell} \sum_{\gamma=0}^{k} v_{i, \gamma} \mathbf{R}_{i, \gamma}^{\top} \mathbf{x}\right] \quad(\bmod q)
\end{aligned}
$$

In the third step of LinFE.Dec we multiply $\mathbf{c}$ with the key e. Recall that by Theorem 3.3 we have $\mathbf{A}_{\vec{v}} \cdot \mathbf{e}=\mathbf{u}(\bmod q)$. It follows that

$$
\mathbf{e}^{\top} \mathbf{c}=\mathbf{u}^{\top} \mathbf{s}+\mathbf{e}^{\top}\left[\mathbf{x} \mid \sum_{i=1}^{\ell} \sum_{\gamma=0}^{k} v_{i, \gamma} \mathbf{R}_{i, \gamma}^{\top} \mathbf{x}\right] \quad(\bmod q) .
$$

Finally, we compute:

$$
\begin{aligned}
z & =c^{\prime}-\mathbf{e}^{\top} \mathbf{c} \quad(\bmod q) \\
& =\left(\mathbf{u}^{\top} \mathbf{s}+x+M \cdot\lfloor q / 2\rceil\right)-\mathbf{u}^{\top} \mathbf{s}-\mathbf{e}^{\top}\left[\mathbf{x} \mid \sum_{i=1}^{\ell} \sum_{\gamma=0}^{k} v_{i, \gamma} \mathbf{R}_{i, \gamma}^{\top} \mathbf{x}\right] \quad(\bmod q) \\
& =M \cdot\lfloor q / 2\rceil+\underbrace{\left(x-\mathbf{e}^{\top}\left[\mathbf{x} \mid \sum_{i=1}^{\ell} \sum_{\gamma=0}^{k} v_{i, \gamma} \mathbf{R}_{i, \gamma}^{\top} \mathbf{x}\right]\right)}_{\text {low-norm noise }}(\bmod q)
\end{aligned}
$$

To obtain $\tilde{M}=M$, it suffices to set the parameters so that with overwhelming probability,

$$
\left|x-\mathbf{e}^{\top}\left[\mathbf{x} \mid \sum_{i=1}^{\ell} \sum_{\gamma=0}^{k} v_{i, \gamma} \mathbf{R}_{i, \gamma}^{\top} \mathbf{x}\right]\right|<q / 4 .
$$


Writing $\mathbf{e}=\left[\mathbf{e}_{1} \mid \mathbf{e}_{2}\right]$ with $\mathbf{e}_{i} \in \mathbb{Z}^{m}$ allows us to rewrite this "noise" term as

$$
x-\left(\mathbf{e}_{1}+\sum_{i=1}^{\ell} \sum_{\gamma=0}^{k} v_{i, \gamma} \mathbf{R}_{i, \gamma} \mathbf{e}_{2}\right)^{\top} \mathbf{x}
$$

By Theorem 3.3 and Lemma 3.2 we have $\|\mathbf{e}\|<\sigma \sqrt{2 m}$ with overwhelming probability. By [3, Lemma 15], we have $\left\|\mathbf{R}_{i, \gamma} \cdot \mathbf{e}_{2}\right\| \leq 12 \sqrt{2 m} \cdot\left\|\mathbf{e}_{2}\right\|$ with overwhelming probability. Since $v_{i, \gamma} \in\{0,1\}$ it follows that

$$
\left\|\mathbf{e}_{1}+\sum_{i=1}^{\ell} \sum_{\gamma=0}^{k} v_{i, \gamma} \mathbf{R}_{i, \gamma} \mathbf{e}_{2}\right\|<(1+12 \cdot \ell \cdot(1+k) \cdot \sqrt{2 m}) \cdot \sigma \sqrt{2 m}=O(\ell \cdot k \cdot \sigma \cdot m) .
$$

It now follows from Lemma 3.9 that the error term (4.2) has absolute value at most

$$
(q \alpha \cdot \omega(\sqrt{\log m})+\sqrt{m} / 2) \cdot O(\ell \cdot \sigma \cdot m \cdot \lg q) .
$$

(Recall that $k=\lfloor\lg q\rfloor$.) For the quantity (4.4) to have absolute value less than $q / 4$, it suffices to choose $q$ and $\alpha$ as in the statement of the Lemma.

\subsection{Security}

We use the simulation technique of Agrawal, Boneh, and Boyen [3] to reduce the security of our system to the hardness of the decision-LWE problem.

Theorem 4.2. Suppose $m \geq 6 n \log q$. If the decision-LWE ${ }_{q, \alpha}$ problem is infeasible, then the predicate encryption scheme described above is weakly attribute hiding.

To prove the theorem we define a series of three games against an adversary $\mathcal{A}$ that plays the weak attribute hiding game (subject to the modification described in Remark 2.3). The adversary $\mathcal{A}$ outputs two attribute vectors $\vec{w}_{0}$ and $\vec{w}_{1}$ at the beginning of each game, and at some point outputs two messages $M_{0}$ and $M_{1}$. Each game comes in two variants, reflecting the choice of attribute/message pair used to create the challenge ciphertext. The first game corresponds to the real security game. In the other two games we use "alternative" setup, key generation, and encryption algorithms Sim.Setup, Sim.KeyGen, and Sim.Enc. The algorithm Sim.Setup takes as additional input an attribute vector $\vec{w}^{*}$, and Sim.Enc takes as additional input the master key output by Sim.Setup. Recall that during the course of the game the adversary can only request keys for predicate vectors $\vec{v}$ such that $\left\langle\vec{v}, \vec{w}_{0}\right\rangle \neq 0$ and $\left\langle\vec{v}, \vec{w}_{1}\right\rangle \neq 0$.

Game $_{0, b}:$ For $b \in\{0,1\}$, the challenger runs the LinFE.Setup algorithm, answers the adversary's secret key queries using the LinFE.KeyGen algorithm, and generates the challenge ciphertext using the LinFE.Enc algorithm with attribute $\vec{w}_{b}$ and message $M_{b}$. 
Game $_{1, b}:$ For $b \in\{0,1\}$, the challenger runs the Sim.Setup algorithm with $\vec{w}^{*}=\vec{w}_{b}$ and answers the adversary's secret key queries using the Sim.KeyGen algorithm. The challenger generates the challenge ciphertext using the Sim.Enc algorithm with attribute $\vec{w}_{b}$ and message $M_{b}$.

$\mathrm{Game}_{2, b}$ : This game is the same as $\mathrm{Game}_{1, b}$, except the challenger generates the challenge ciphertext by choosing a uniformly random element of the ciphertext space.

We now define the alternative setup, key generation, and encryption algorithms.

$\operatorname{Sim}$.Setup $\left(1^{n}, 1^{\ell}, \vec{w}^{*}\right)$ : On input a security parameter $n$, a parameter $\ell$ denoting the dimension of predicate and attribute vectors, and an attribute vector $\vec{w}^{*} \in \mathbb{Z}_{q}^{\ell}$, do the following:

1. Choose a random matrix $\mathbf{A} \stackrel{\mathrm{R}}{\leftarrow} \mathbb{Z}_{q}^{n \times m}$ and a random vector $\mathbf{u} \stackrel{\mathrm{R}}{\leftarrow} \mathbb{Z}_{q}^{n}$.

2. Use $\operatorname{TrapGen}(q, n, m)$ to generate a matrix $\mathbf{B}^{*} \in \mathbb{Z}_{q}^{n \times m}$ along with a basis $\mathbf{T}_{\mathbf{B}^{*}}$ of $\Lambda_{q}^{\perp}\left(\mathbf{B}^{*}\right)$.

3. For $i=1, \ldots, \ell$ and $\gamma=0, \ldots, k$, pick random matrices $\mathbf{R}_{i, \gamma}^{*} \stackrel{\mathrm{R}}{\leftarrow}\{-1,1\}^{m \times m}$ and set

$$
\mathbf{A}_{i, \gamma} \leftarrow \mathbf{A} \mathbf{R}_{i, \gamma}^{*}-2^{\gamma} w_{i}^{*} \mathbf{B}^{*}
$$

Output the public parameters and master key

$\mathrm{PP}=\left(\mathbf{A},\left\{\mathbf{A}_{i, \gamma}\right\}_{i \in\{1, \ldots, \ell\}, \gamma \in\{0, \ldots, k\}}, \mathbf{u}\right), \quad \mathbf{M K}=\left(\vec{w}^{*},\left\{\mathbf{R}_{i, \gamma}^{*}\right\}_{i \in\{1, \ldots, \ell\}, \gamma \in\{0, \ldots, k\}}, \mathbf{B}^{*}, \mathbf{T}_{\mathbf{B}^{*}}\right)$

Sim.KeyGen(PP, MK, $\vec{v})$ : On input public parameters PP, a master key MK, and a vector $\vec{v} \in \mathbb{Z}_{q}^{\ell}$, do the following:

1. If $\left\langle\vec{v}, \vec{w}^{*}\right\rangle=0$, output $\perp$.

2. Define the binary decomposition of $v_{i}$ as in 4.1.

3. Define the matrices

$$
\mathbf{C}_{\vec{v}}:=\sum_{i=1}^{\ell} \sum_{\gamma=0}^{k} v_{i, \gamma} \mathbf{A}_{i, \gamma} \in \mathbb{Z}_{q}^{n \times m}, \quad \mathbf{A}_{\vec{v}}:=\left[\mathbf{A} \| \mathbf{C}_{\vec{v}}\right] \in \mathbb{Z}_{q}^{n \times 2 m} .
$$

Observe that

$$
\mathbf{A}_{\vec{v}}=[\mathbf{A} \| \mathbf{A}\left(\sum_{i=1}^{\ell} \sum_{\gamma=0}^{k} v_{i, \gamma} \mathbf{R}_{i, \gamma}^{*}\right)-\underbrace{\left(\sum_{i=1}^{\ell} \sum_{\gamma=0}^{k} 2^{\gamma} v_{i, \gamma} w_{i}^{*}\right)}_{\left\langle\vec{v}, \vec{w}^{*}\right\rangle} \mathbf{B}^{*}] .
$$

4. Let $\mathbf{e} \leftarrow$ SampleRight $\left(\mathbf{A},-\left\langle\vec{v}, \vec{w}^{*}\right\rangle \mathbf{B}^{*}, \sum_{i=1}^{\ell} \sum_{\gamma=0}^{k} v_{i, \gamma} \mathbf{R}_{i, \gamma}^{*}, \mathbf{T}_{\mathbf{B}^{*}}, \mathbf{u}, \sigma\right) \in$ $\mathbb{Z}_{q}^{2 m}$

Output the secret key $\mathrm{sk}_{\vec{v}}=\mathbf{e}$. 
Sim.Enc(PP, $\vec{w}, M, \mathrm{MK})$ : This algorithm is the same as the LinFE.Enc algorithm, except:

1. In Step 1, matrix $\mathbf{B}^{*} \in M K$ is used instead of a random matrix $\mathbf{B}$.

2. In Step 5a, the matrices $\mathbf{R}_{i, \gamma}^{*} \in \mathrm{MK}$ for are used instead of random matrices $\mathbf{R}_{i, \gamma}$ for $i=1, \ldots, \ell$ and $\gamma=0, \ldots, k$.

To prove security of our system, we show that the two games in each of the pairs $\left(\mathrm{Game}_{0, b}, \mathrm{Game}_{1, b}\right)$, $\left(\mathrm{Game}_{1, b}, \mathrm{Game}_{2, b}\right)$ and $\left(\mathrm{Game}_{2,0}, \mathrm{Game}_{2,1}\right)$ are either statistically or computationally indistinguishable (under the decision-LWE assumption) from the point of view of the adversary. Theorem 4.2 then follows from a simple hybrid argument; details are in the full version of this paper [2].

Lemma 4.3. For a given $b \in\{0,1\}$, the view of the adversary $\mathcal{A}$ in $\mathrm{Game}_{0, b}$ is statistically close to the view of $\mathcal{A}$ in $\mathrm{Game}_{1, b}$.

The proof of Lemma 4.3 can be found in the full version of this paper [2].

Lemma 4.4. For a given $b \in\{0,1\}$, if the decision-LWE assumption holds, then the view of the adversary $\mathcal{A}$ in $\mathrm{Game}_{1, b}$ is computationally indistinguishable from the view of $\mathcal{A}$ in Game $_{2, b}$.

Proof. Suppose we are given $m+1$ LWE challenges $\left(\mathbf{a}_{i}, y_{i}\right) \in \mathbb{Z}_{q}^{n} \times \mathbb{Z}_{q}$ for $j=$ $0, \ldots, m$, where either $y_{j}=\left\langle\mathbf{a}_{j}, \mathbf{s}\right\rangle+x_{j}$ for some (fixed) random secret $\mathbf{s} \stackrel{\mathrm{R}}{\leftarrow} \mathbb{Z}_{q}^{n}$ and Gaussian noise $x_{j} \leftarrow \bar{\Psi}_{\alpha}$, or $y_{j}$ is uniformly random in $\mathbb{Z}_{q}$ (and this choice is the same for each challenge). We define the following variables:

$$
\begin{aligned}
& \mathbf{A}:=\left(\begin{array}{ccc}
\mid & & \mid \\
\mathbf{a}_{1} & \cdots & \mathbf{a}_{m} \\
\mid & & \mid
\end{array}\right) \in \mathbb{Z}_{q}^{n \times m} \mathbf{u}:=\mathbf{a}_{0} \\
& \mathbf{c}_{0}:=\left(y_{1}, \ldots, y_{m}\right) \in \mathbb{Z}_{q}^{m} \quad c^{\prime}:=y_{0}+M_{b} \cdot\left\lfloor\frac{q}{2}\right\rceil
\end{aligned}
$$

We simulate the challenger as follows:

- Setup: Run Sim.Setup with $\vec{w}^{*}=\vec{w}_{b}$, and let $\mathbf{A}$ and $\mathbf{u}$ be as in (4.5).

- Private key queries: Run the Sim.KeyGen algorithm.

- Challenge ciphertext: For $i=1, \ldots, \ell$ and $\gamma=0, \ldots, k$, let $\mathbf{c}_{i, \gamma}=\mathbf{R}_{i, \gamma}^{*}{ }^{\top} \mathbf{c}_{0}$ (using $\mathbf{R}_{i, \gamma}^{*} \in \mathrm{MK}$ ). Output $\left(\mathbf{c}_{0},\left\{\mathbf{c}_{i, \gamma}\right\}_{i \in\{1, \ldots, \ell\}, \gamma \in\{0, \ldots, k\}}, c^{\prime}\right)$.

Now observe that for $i=1, \ldots, \ell$ and $\gamma=0, \ldots, k$, the Sim.Enc algorithm sets

$$
\mathbf{c}_{i, \gamma}=\left(\mathbf{A} \mathbf{R}_{i, \gamma}-2^{\gamma} w_{i}^{*} \mathbf{B}^{*}+2^{\gamma} w_{i}^{*} \mathbf{B}^{*}\right)^{\top} \mathbf{s}+\mathbf{R}_{i, \gamma}^{*}{ }^{\top} \mathbf{x}=\mathbf{R}_{i, \gamma}^{*}{ }^{\top}\left(\mathbf{A}^{\top} \mathbf{s}+\mathbf{x}\right) .
$$

It follows that if $y_{j}=\left\langle\mathbf{a}_{j}, \mathbf{s}\right\rangle+x_{j}$, then $\mathbf{c}_{i, \gamma}=\mathbf{R}_{i, \gamma}^{*}{ }^{\top} \mathbf{c}_{0}$ and the simulator described above is identical to the challenger in Game $\mathrm{G}_{1, b}$.

On the other hand, if $y_{j}$ is random in $\mathbb{Z}_{q}$, then the simulated ciphertext is $\left(\mathbf{c}_{0}, \overline{\mathbf{R}}^{\top} \mathbf{c}_{0}, c^{\prime}\right)$, where $\overline{\mathbf{R}^{*}}$ is the concatenation of the matrices $\mathbf{R}_{i, \gamma}^{*}$. By the standard leftover hash lemma (e.g. [43, Theorem 8.37]), the quantities $\mathbf{A} \overline{\mathbf{R}^{*}}$ and $\overline{\mathbf{R}}^{\top} \mathbf{c}_{0}$ are 
independent uniformly random samples. Thus in this case the ciphertext is uniformly random and the simulator described above is identical the challenger in Game $\mathrm{G}_{2, b}$.

We conclude that any efficient adversary that can distinguish Game ${ }_{1, b}$ from Game $\mathrm{G}_{2, b}$ can solve the decision-LWE problem.

Lemma 4.5. The view of the adversary $\mathcal{A}$ in $\mathrm{Game}_{2,0}$ is statistically indistinguishable from the view of $\mathcal{A}$ in $\mathrm{Game}_{2,1}$.

Proof. Note that the only place where $\vec{w}^{*}$ appears in Game ${ }_{2, b}$ is in the public parameter $\mathbf{A}_{i, \gamma}:=\mathbf{A R}_{i, \gamma}^{*}-2^{\gamma} w_{i}^{*} \mathbf{B}^{*}$. Let $\overline{\mathbf{A}} \in \mathbb{Z}_{q}^{n \times m \ell(k+1)}$ and $\overline{\mathbf{R}^{*}} \in \mathbb{Z}_{q}^{m \times m \ell(k+1)}$ be the concatenations of the $\mathbf{A}_{i, \gamma}$ and the $\mathbf{R}_{i, \gamma}^{*}$, respectively. Then we have $\overline{\mathbf{A}}=\mathbf{A} \overline{\mathbf{R}^{*}}$. By [3, Lemma 13] the pair $\left(\mathbf{A}, \mathbf{A} \overline{\mathbf{R}}^{*}\right)$ is statistically indistinguishable from $(\mathbf{A}, \mathbf{C})$ where $\mathbf{C}$ is uniformly random. Since for any fixed value of $\mathbf{X}$ and uniformly random $\mathbf{C}$, the variable $\mathbf{C}-\mathbf{X}$ is also uniformly random, it follows that the distributions of $\mathbf{A}_{i, \gamma}$ in the two games are statistically indistinguishable.

\subsection{Parameter Selection}

We can extract from the above description the parameters required for correctness and security of the system. For correctness of decryption, by Lemma 4.1 we require

$$
q / \lg q=\Omega\left(\sigma \cdot \ell \cdot m^{3 / 2}\right) \quad \text { and } \quad \alpha \leq(\log q \cdot \sigma \cdot \ell \cdot m \cdot \omega \sqrt{\log m})^{-1}
$$

In our security theorem (Theorem 4.2), we require $m>6 n \lg q$ in order for the output of TrapGen to be statistically random. The additional constraints imposed by our security reduction are the following:

- From the description of LinFE.Setup and LinFE.KeyGen, we have $\left\|\widetilde{\mathbf{T}_{\mathbf{A}}}\right\|=$ $O(\sqrt{n \log q})$ (by Theorem 3.1) and $\mathbf{e} \leftarrow \mathcal{D}_{\Lambda_{q}^{\mathrm{u}}\left(\mathbf{A}_{\vec{v}}\right), \sigma}$ (by Theorem 3.3), subject to the requirement that

$$
\sigma \geq\left\|\widetilde{\mathbf{T}}_{\mathbf{A}}\right\| \cdot \omega(\sqrt{\log m})=O(\sqrt{n \log q}) \cdot \omega(\sqrt{\log m}) .
$$

- From the description of Sim.Setup and Sim.KeyGen, we have $\left\|\widetilde{\mathbf{T}_{\mathbf{B}^{*}}}\right\|=$ $O(\sqrt{n \log q})$ (by Theorem 3.1), and $\mathbf{e} \leftarrow \mathcal{D}_{\Lambda_{q}^{\mathrm{u}}\left(\mathbf{A}_{\vec{v}}\right), \sigma}$ (by Theorem 3.4), subject to the requirement that

$$
\sigma \geq\left\|\widetilde{\mathbf{T}}_{\mathbf{B}^{*}}\right\| \cdot s_{R} \cdot \omega(\sqrt{\log m})
$$

Since $\mathbf{R}$ is a sum of $\ell \cdot(\lg q+1)$ random matrices with $\{1,-1\}$ entries, it follows from [3, Lemma 15] that $s_{R}=\sup _{\{\mathbf{x}:\|\mathbf{x}\|=1\}}\|\mathbf{R x}\|=O(\ell \cdot(\lg q+1) \cdot \sqrt{m})$ with overwhelming probability. Plugging this value into 4.7), we see that it suffices to choose

$$
\sigma \geq O(\sqrt{n \log q}) \cdot O(\ell \cdot(\lg q+1) \cdot \sqrt{m}) \cdot \omega(\sqrt{\log m}) .
$$


Thus to satisfy the more stringent of the above two conditions (i.e., the latter), we set

$$
\sigma=\omega(m \cdot \ell \cdot \log q \cdot \sqrt{\log m})
$$

using the fact (noted above) that $m \geq 6 n \log q$.

In order to reduce decision-LWE to approximating worst-case lattice problems to within $\operatorname{poly}(n)$ factors we have two options: for polynomial-size $q$ we can use Regev's quantum reduction (Theorem 3.7) with $q \alpha>2 \sqrt{n}$ and $\alpha \geq 1 / \operatorname{poly}(n)$, while for exponential-size $q$ we can use Peikert's classical reduction (Theorem 3.8) with each prime factor $q_{i}$ of $q$ satisfying $\omega(\sqrt{\log n}) / \alpha<q_{i}<\operatorname{poly}(n)$. (Note that a large value of $q$ may be required for certain applications; see the full version of this paper [2, $\S 5]$ for details.)

The following selection of parameters satisfies all of these constraints. For a given $\ell$, pick a small constant $\delta>0$, and set

$$
\begin{aligned}
m & =\left\lceil n^{1+\delta}\right\rceil, & & \text { to satisfy } m>6 n \lg q \\
\sigma & =\left\lceil n^{2+2 \delta} \cdot \ell\right\rceil, & & \text { to satisfy 4.8 } \\
q_{i} & =\text { the } i \text { th prime larger than }(\ell \log \ell)^{2} \cdot n^{7 / 2+5 \delta} & & \\
\alpha & =\Omega\left((\ell \log \ell)^{2} \cdot n^{3+5 \delta}\right)^{-1} & & \text { to satisfy 4.6 }
\end{aligned}
$$

Observe that the above setting of parameters satisfies the conditions for applying Theorems 3.7 and 3.8. To obtain polynomial size $q$ we use $q=q_{1}$, while to obtain exponential size $q$ we use $q=\prod_{i=1}^{\tau} q_{i}$, where $\tau$ is chosen so that $q>2^{n / 2}$. In either case we can choose $\delta$ large enough so that $n^{1+\delta}>6 n \lg q$. In the former case, the security of the scheme can be based on the hardness of approximating SIVP and GapSVP to within a factor of $\tilde{O}(n / \alpha)=\tilde{O}\left((\ell \log \ell)^{2} \cdot n^{4+5 \delta}\right)$ in the worst case (by quantum algorithms). In the latter case, security is based on the hardness of approximating GapSVP to within a factor of $\tilde{O}(n / \alpha)=\tilde{O}\left((\ell \log \ell)^{2} \cdot n^{4+5 \delta}\right)$ in the worst case (by classical algorithms).

Note that since $m>n \lg q$ and $q_{i}>n$, the matrices $\mathbf{A}$ and $\mathbf{B}$ have full rank modulo each prime divisor of $q$ with overwhelming probability, as required for successful execution of the SampleLeft and SampleRight algorithms.

Finally, we note that these parameter choices are not necessarily optimal, and one might be able to set the parameters to have somewhat smaller values while maintaining correctness and security. In particular, one might be able to reduce the ciphertext size by using the $r$-ary expansion of the vector $\vec{v}$ for some $r>2$ instead of the binary expansion as described above.

\section{Conclusion and Open Questions}

We have presented a lattice-based predicate encryption scheme for inner product predicates whose security follows from the difficulty of the learning with errors problem. Our construction can instantiate applications such as range and subset queries, polynomial evaluation, and CNF/DNF formulas on encrypted data. (A more detailed discussion of these applications appears in the full version of this paper [2].) Our construction is the first functional encryption scheme based on lattice techniques that goes beyond basic identity-based encryption. 
Many open questions still remain in this field. One direction of research is to improve the security of our construction. Our scheme is weakly attribute hiding in the selective security model, but for stronger security guarantees we would like to construct a scheme that is fully secure and/or fully attribute hiding. Achieving either task will require new simulation techniques; a natural question is whether the "dual-system" approach introduced by Waters [45] and used to prove full security of attribute-based encryption and predicate encryption constructions using bilinear groups [30,9,34] can be adapted to lattice-based constructions.

Another direction of research is to improve the efficiency of our scheme. If $q=2^{O(n)}$ is exponential size, as is needed for several of our applications, then setting the parameters as recommended in Section 4.4 gives public parameters of size $\Theta\left(\ell n m \lg ^{2}(q)\right)=\Omega\left(\ell n^{5}\right)$ and ciphertexts of size $\Theta\left(\ell m \lg ^{2}(q)\right)=\Omega\left(\ell n^{4}\right)$, which may be too large for practical purposes. A construction that achieved the same functionality with polynomial-size $q$ would be a significant step forward. The ring-LWE problem introduced by Lyubashevsky, Peikert, and Regev [31] seems to be a natural candidate for such a construction.

Finally, it is a open question to construct predicate encryption schemes (via any technique) that support a greater range of functionality than inner product predicates. Ideally we would like a system that could support any polynomial-size predicate on encrypted data. Now that predicate encryption has moved into the world of lattices, perhaps techniques used to construct fully homomorphic encryption from lattices [23, 19, 18] could be used to help us move towards this goal.

Acknowledgments. The authors thank Dan Boneh, Brent Waters, Hoeteck Wee, and the anonymous referees for helpful discussions and comments.

\section{References}

1. Abdalla, M., Bellare, M., Catalano, D., Kiltz, E., Kohno, T., Lange, T., Malone-Lee, J., Neven, G., Paillier, P., Shi, H.: Searchable encryption revisited: Consistency properties, relation to anonymous IBE, and extensions. J. Cryptology 21(3), 350-391 (2008)

2. Agrawal, S., Freeman, D.M., Vaikuntanathan, V.: Functional encryption for inner product predicates from learning with errors. Cryptology ePrint Report 2011/410 (2011) (full version of this paper), http://eprint.iacr.org/2011/410

3. Agrawal, S., Boneh, D., Boyen, X.: Efficient Lattice (H)IBE in the Standard Model. In: Gilbert, H. (ed.) EUROCRYPT 2010. LNCS, vol. 6110, pp. 553-572. Springer, Heidelberg (2010), full version at, http://crypto.stanford.edu/ dabo/pubs/papers/latticebb.pdf

4. Agrawal, S., Boneh, D., Boyen, X.: Lattice Basis Delegation in Fixed Dimension and ShorterCiphertext Hierarchical IBE. In: Rabin, T. (ed.) CRYPTO 2010. LNCS, vol. 6223, pp. 98115. Springer, Heidelberg (2010)

5. Agrawal, S., Boyen, X.: Identity-based encryption from lattices in the standard model (July 2009) (manuscript), http: / / www.cs.stanford.edu/ xb/ab09/

6. Ajtai, M.: Generating Hard Instances of the Short Basis Problem. In: Wiedermann, J., Van Emde Boas, P., Nielsen, M. (eds.) ICALP 1999. LNCS, vol. 1644, pp. 1-9. Springer, Heidelberg (1999)

7. Alwen, J., Peikert, C.: Generating shorter bases for hard random lattices. In: STACS, pp. 7586 (2009), full version available at http://www.cc.gatech.edu/ cpeikert/pubs/shorter.pdf 
8. Attrapadung, N., Imai, H.: Conjunctive Broadcast and Attribute-Based Encryption. In: Shacham, H., Waters, B. (eds.) Pairing 2009. LNCS, vol. 5671, pp. 248-265. Springer, Heidelberg (2009)

9. Attrapadung, N., Libert, B.: Functional Encryption for Inner Product: Achieving ConstantSize Ciphertexts with Adaptive Security or Support for Negation. In: Nguyen, P.Q., Pointcheval, D. (eds.) PKC 2010. LNCS, vol. 6056, pp. 384-402. Springer, Heidelberg (2010)

10. Bethencourt, J., Sahai, A., Waters, B.: Ciphertext-policy attribute-based encryption. In: IEEE Symposium on Security and Privacy, pp. 321-334 (2007)

11. Blundo, C., Iovino, V., Persiano, G.: Predicate Encryption with Partial Public Keys. In: Heng, S.H., Wright, R.N., Goi, B.M. (eds.) CANS 2010. LNCS, vol. 6467, pp. 298-313. Springer, Heidelberg (2010)

12. Boneh, D., Boyen, X.: Efficient Selective-ID Secure Identity-Based Encryption Without Random Oracles. In: Cachin, C., Camenisch, J. (eds.) EUROCRYPT 2004. LNCS, vol. 3027, pp. 223-238. Springer, Heidelberg (2004)

13. Boneh, D., Boyen, X.: Secure Identity Based Encryption Without Random Oracles. In: Franklin, M. (ed.) CRYPTO 2004. LNCS, vol. 3152, pp. 443-459. Springer, Heidelberg (2004)

14. Boneh, D., Franklin, M.: Identity-based encryption from the Weil pairing. SIAM J. Comput. 32(3), 586-615 (2003); extended abstract in CRYPTO 2001

15. Boneh, D., Di Crescenzo, G., Ostrovsky, R., Persiano, G.: Public Key Encryption with Keyword Search. In: Cachin, C., Camenisch, J. (eds.) EUROCRYPT 2004. LNCS, vol. 3027, pp. 506-522. Springer, Heidelberg (2004)

16. Boneh, D., Sahai, A., Waters, B.: Functional Encryption: Definitions and Challenges. In: Ishai, Y. (ed.) TCC 2011. LNCS, vol. 6597, pp. 253-273. Springer, Heidelberg (2011)

17. Boneh, D., Waters, B.: Conjunctive, Subset, and Range Queries on Encrypted Data. In: Vadhan, S.P. (ed.) TCC 2007. LNCS, vol. 4392, pp. 535-554. Springer, Heidelberg (2007)

18. Brakerski, Z., Vaikuntanathan, V.: Efficient fully homomorphic encryption from (standard) LWE. To appear in FOCS 2011 (2011), preprint available at http: / / eprint.iacr.org/2011/344

19. Brakerski, Z., Vaikuntanathan, V.: Fully Homomorphic Encryption from Ring-LWE and Security for Key Dependent Messages. In: Rogaway, P. (ed.) CRYPTO 2011. LNCS, vol. 6841, pp. 505-524. Springer, Heidelberg (2011)

20. Cash, D., Hofheinz, D., Kiltz, E., Peikert, C.: Bonsai Trees, or How to Delegate a Lattice Basis. In: Gilbert, H. (ed.) EUROCRYPT 2010. LNCS, vol. 6110, pp. 523-552. Springer, Heidelberg (2010)

21. Chase, M.: Multi-Authority Attribute Based Encryption. In: Vadhan, S.P. (ed.) TCC 2007. LNCS, vol. 4392, pp. 515-534. Springer, Heidelberg (2007)

22. Gentry, C.: Practical Identity-Based Encryption Without Random Oracles. In: Vaudenay, S. (ed.) EUROCRYPT 2006. LNCS, vol. 4004, pp. 445-464. Springer, Heidelberg (2006)

23. Gentry, C.: A fully homomorphic encryption scheme. Ph.D. thesis, Stanford University (2009), http: / / crypto.stanford.edu/craig

24. Gentry, C., Peikert, C., Vaikuntanathan, V.: Trapdoors for hard lattices and new cryptographic constructions. In: 40th ACM Symposium on Theory of Computing — STOC 2008, pp. 197206. ACM (2008)

25. Gentry, C., Silverberg, A.: Hierarchical ID-Based Cryptography. In: Zheng, Y. (ed.) ASIACRYPT 2002. LNCS, vol. 2501, pp. 548-566. Springer, Heidelberg (2002)

26. Goyal, V., Jain, A., Pandey, O., Sahai, A.: Bounded Ciphertext Policy Attribute Based Encryption. In: Aceto, L., Damgård, I., Goldberg, L.A., Halldórsson, M.M., Ingólfsdóttir, A., Walukiewicz, I. (eds.) ICALP 2008, Part II. LNCS, vol. 5126, pp. 579-591. Springer, Heidelberg (2008)

27. Goyal, V., Pandey, O., Sahai, A., Waters, B.: Attribute-based encryption for fine-grained access control of encrypted data. In: ACM Conference on Computer and Communications Security, pp. 89-98 (2006) 
28. Gordon, S.D., Katz, J., Vaikuntanathan, V.: A Group Signature Scheme from Lattice Assumptions. In: Abe, M. (ed.) ASIACRYPT 2010. LNCS, vol. 6477, pp. 395-412. Springer, Heidelberg (2010)

29. Katz, J., Sahai, A., Waters, B.: Predicate Encryption Supporting Disjunctions, Polynomial Equations, and Inner Products. In: Smart, N. (ed.) EUROCRYPT 2008. LNCS, vol. 4965, pp. 146-162. Springer, Heidelberg (2008), http: / / eprint.iacr.org/2007/404

30. Lewko, A., Okamoto, T., Sahai, A., Takashima, K., Waters, B.: Fully Secure Functional Encryption: Attribute-Based Encryption and (Hierarchical) Inner Product Encryption. In: Gilbert, H. (ed.) EUROCRYPT 2010. LNCS, vol. 6110, pp. 62-91. Springer, Heidelberg (2010), full version available at http: / / eprint.iacr.org/2010/110

31. Lyubashevsky, V., Peikert, C., Regev, O.: On Ideal Lattices and Learning with Errors Over Rings. In: Gilbert, H. (ed.) EUROCRYPT 2010. LNCS, vol. 6110, pp. 1-23. Springer, Heidelberg (2010)

32. Micciancio, D., Regev, O.: Worst-case to average-case reductions based on Gaussian measures. In: 45th IEEE Symposium on Foundations of Computer Science - FOCS 2004, pp. 372-381 (2004)

33. Okamoto, T., Takashima, K.: Hierarchical Predicate Encryption for Inner-Products. In: Matsui, M. (ed.) ASIACRYPT 2009. LNCS, vol. 5912, pp. 214-231. Springer, Heidelberg (2009)

34. Okamoto, T., Takashima, K.: Fully Secure Functional Encryption with General Relations from the Decisional Linear Assumption. In: Rabin, T. (ed.) CRYPTO 2010. LNCS, vol. 6223, pp. 191-208. Springer, Heidelberg (2010)

35. Ostrovsky, R., Sahai, A., Waters, B.: Attribute-based encryption with non-monotonic access structures. In: ACM Conference on Computer and Communications Security, pp. 195-203 (2007)

36. Peikert, C.: Public-key cryptosystems from the worst-case shortest vector problem. In: 41st Annual ACM Symposium on Theory of Computing - STOC 2009, pp. 333-342 (2009)

37. Peikert, C.: An Efficient and Parallel Gaussian Sampler for Lattices. In: Rabin, T. (ed.) CRYPTO 2010. LNCS, vol. 6223, pp. 80-97. Springer, Heidelberg (2010)

38. Regev, O.: On lattices, learning with errors, random linear codes, and cryptography. In: 37th Annual ACM Symposium on Theory of Computing — STOC 2005, pp. 84-93 (2005)

39. Sahai, A., Waters, B.: Fuzzy Identity-Based Encryption. In: Cramer, R. (ed.) EUROCRYPT 2005. LNCS, vol. 3494, pp. 457-473. Springer, Heidelberg (2005)

40. Shen, E., Shi, E., Waters, B.: Predicate Privacy in Encryption Systems. In: Reingold, O. (ed.) TCC 2009. LNCS, vol. 5444, pp. 457-473. Springer, Heidelberg (2009)

41. Shi, E., Bethencourt, J., Chan, H.T.H., Song, D.X., Perrig, A.: Multi-dimensional range query over encrypted data. In: IEEE Symposium on Security and Privacy, pp. 350-364 (2007)

42. Shi, E., Waters, B.: Delegating Capabilities in Predicate Encryption Systems. In: Aceto, L., Damgård, I., Goldberg, L.A., Halldórsson, M.M., Ingólfsdóttir, A., Walukiewicz, I. (eds.) ICALP 2008, Part II. LNCS, vol. 5126, pp. 560-578. Springer, Heidelberg (2008)

43. Shoup, V.: A Computational Introduction to Number Theory and Algebra, 2nd edn. Cambridge University Press (2008)

44. Waters, B.: Efficient Identity-Based Encryption Without Random Oracles. In: Cramer, R. (ed.) EUROCRYPT 2005. LNCS, vol. 3494, pp. 114-127. Springer, Heidelberg (2005)

45. Waters, B.: Dual System Encryption: Realizing Fully Secure IBE and HIBE Under Simple Assumptions. In: Halevi, S. (ed.) CRYPTO 2009. LNCS, vol. 5677, pp. 619-636. Springer, Heidelberg (2009)

46. Waters, B.: Ciphertext-Policy Attribute-Based Encryption: An Expressive, Efficient, and Provably Secure Realization. In: Catalano, D., Fazio, N., Gennaro, R., Nicolosi, A. (eds.) PKC 2011. LNCS, vol. 6571, pp. 53-70. Springer, Heidelberg (2011) 